\title{
Effect of Actuator Torque Degradation on Behavior of a 6-DOF Industrial Robot
}

\author{
Le Ngoc Truc ${ }^{1}$, Nguyen Tuan Nghia ${ }^{2}$, Nguyen Trung Thanh ${ }^{2}$, Nguyen Minh Thien², \\ Tung Lam Nguyen ${ }^{2, *}$ \\ ${ }^{1}$ Institute for Control Engineering and Automation, Hanoi University of Science and Technology, Vietnam \\ ${ }^{2}$ School of Electrical Engineering, Hanoi University of Science and Technology, Vietnam
}

Received February 18, 2020; Revised March 18, 2020; Accepted March 28, 2020

Copyright $\odot 2020$ by authors, all rights reserved. Authors agree that this article remains permanently open access under the terms of the Creative Commons Attribution License 4.0 International License

\begin{abstract}
Actuator faults of robot manipulators may occur during their lifetime after long time in operation. There are several kinds of actuator failures such as locked joints, free-swinging joints, and loss of actuator torque effectiveness. The main goals of this paper are (i) to classify the loss of torque effectiveness, called torque degradation, into three divergent cases: Boundary Degradation of Torque (BDT), Boundary Degradation of Torque Rate (BDTR), and Proportional Degradation of Torque (PDT); and (ii) to analyze their effect on behavior of a typical industrial robot. The possible failures might degrade the whole system performance or in some certain cases leading to unavoidable damages. In normal operation, we do not have a controller designed specifically for these faults. In order to have a better understanding on how the mentioned problems affect robot operations, with an assumption that the knowledge of robot parameters are known, a closed-loop control law is used to demonstrate the control ability in dealing with these cases. By taking advantage of MATLAB/Simscape Multibody, the quasi-physical model of robot is employed instead of expensive prototypes and experiments. Simulation results show that the joint responses according to different types of failures. In many cases, the robot cannot track the reference trajectories properly.
\end{abstract}

Keywords Industrial Robot, Torque Degradation, ActuatorFault, Joint Failure, Simscape Multibody

\section{Introduction}

For many years, researches in the field of robot manipulators have received a large amount of attention, especially in cardinal industries because of the ability to replace people working in hazardous and risky environments with higher flexibility and precision [1]-[4]. The performance and accuracy of the robotic arms may be affected by actuator failures. There have been many studies on the faults of actuators. In those studies, methods to improve the tracking performance of manipulators have introduced, such as [5]-[9]. For example, in [5], the locked joint failure was addressed with matrix perturbation methodology which is extended to deal with multiple locked joint failures. In [6], an effective approach was introduced to guarantee that kinematically redundant manipulators can perform the task after experiencing a locked jo int failure. In [7], the problem of how to optimally readjust a kinematically redundant manipulator in anticipating a free-swinging failure was presented. However, these studies for actuator failures just mentioned locked joint faults or free-swinging joint faults. In practical situations, robotic arms may also be subject to effect of actuator torque degradation faults. Therefore, this actual problem should be considered to suggest approaches to compensate for failures and improve manipulator effectiveness. Presently, there are a significant number of studies that address actuator failures in torque degradation, such as [10]-[18]. In [10], the author formu lated a method for fault compensation based on the initiative of a corrective control law which is enabled when a fault occurs. Nevertheless, the diffe rent categories of torque degradation faults were not mentioned. In [11], a novel event-triggered control method for a class of uncertain nonlinear systems suffering unknown actuator failures was indicated. A novel adaptive fault accommodation control approach is nominated for para metric strict feedback nonlinear systems [12]. In [13], the authors presented a new adaptive fault tolerant controller design for unknown nonlinear systems with multiple actuators. In [15], a kinematic fault tolerant control for robotic arms which are subject to actuator failures is proposed. Several tests are effectuated on two types of actuator failures: additive time-varying fault and 
additive constant fault. In [16], a model-based fault detection and isolation method for robotic arms based on adaptive nonlinear observer was proposed to deal with two cases: actuator and sensor faults. The results in [17] proposed a failure detection and adaptive compensation control approach for flexible-joint manipulators with model uncertainties and multip le actuator failures. In [11][18], the actuators operating in a fault mode were modeled. The Proportional Degradation of Torque (PDT) and the failure where actuators whose torque are stuck at an unknown value were mentioned as well. But, the Boundary Degradation of Torque (BDT) and the Boundary Degradation of Torque Rate (BDTR) did not analy ze yet. On the other hand, those studies in [11]-[14] conducted on general nonlinear systems. A study on the effect of actuator torque degradation faults of a six degree of freedom (6-DOF) manipulator has considerable differences.

In this paper, the actuator torque degradation faults of the 6-DOF manipulator are divided into three cases: BDT, BDTR, and PDT. A backstepping controller is applied to robot IRB 120 which is experienced the above failures to evaluate the control ability. To enhance the accuracy and reliability of simulation, robot IRB 120 is modeled by Simscape Multibody. The rest of this paper is organized as follows. In section 2, mathematical modeling of the robot and Simscape-based model are denoted. Three cases of actuator torque degradation faults (BDT, BDTR, and PDT) are formulated in section 3. In section 4, a backstepping control methodology is designed for the robot in normal operation. Section 5 presents simulation examples to investigate the performance of robot in case of torque degradation. Finally, some conclusions are drawn in the last section.

\section{Industrial robot IRB 120}

\subsection{Mathematical Modeling of Robot Dynamics}

Robot IRB 120 which is one type of 6-DOF industrial robots manufactured by ABB Corporation has the configuration and D-H parameters described as follows (Figure 1 and Table 1):

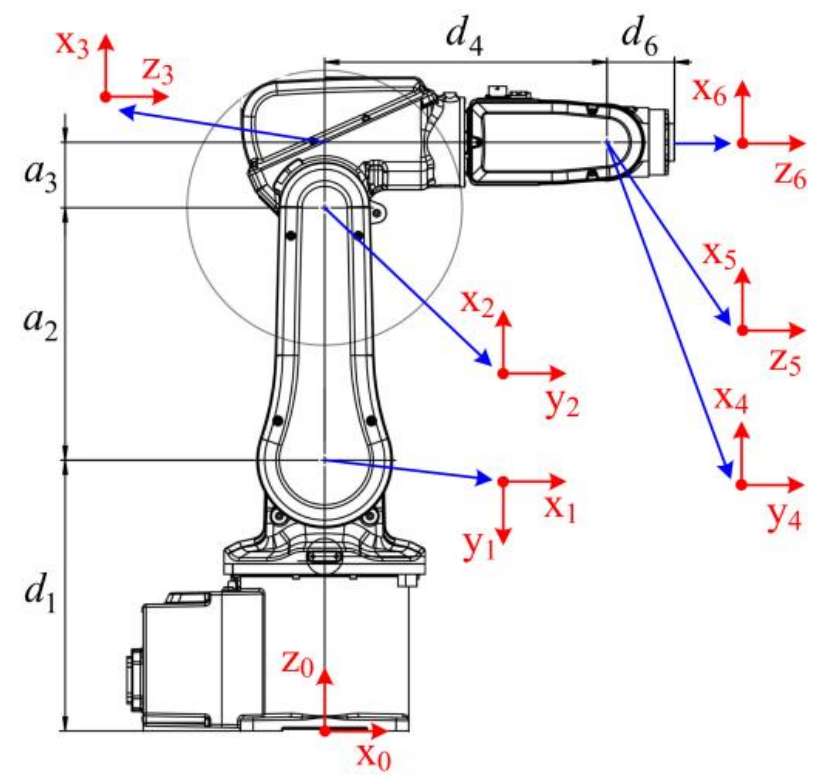

Figure 1. Initial configuration and attached frames of robot IRB 120

Table 1. D-H parameters of robot IRB 120

\begin{tabular}{|c|l|l|l|l|}
\hline Joint $i$ & \multicolumn{1}{|c|}{$\theta_{i}(\mathrm{rad})$} & \multicolumn{1}{|c|}{$d_{i}(\mathrm{~m})$} & \multicolumn{1}{|c|}{$a_{i}(\mathrm{~m})$} & \multicolumn{1}{c|}{$\alpha_{i}(\mathrm{rad})$} \\
\hline 1 & $q_{1}$ & $d_{1}=0.29$ & $a_{1}=0$ & $\alpha_{1}=-\pi / 2$ \\
\hline 2 & $q_{2}-\pi / 2$ & $d_{2}=0$ & $a_{2}=0.27$ & $\alpha_{2}=0$ \\
\hline 3 & $q_{3}$ & $d_{3}=0$ & $a_{3}=0.07$ & $\alpha_{3}=-\pi / 2$ \\
\hline 4 & $q_{4}$ & $d_{4}=0.302$ & $a_{4}=0$ & $\alpha_{4}=\pi / 2$ \\
\hline 5 & $q_{5}$ & $d_{5}=0$ & $a_{5}=0$ & $\alpha_{5}=-\pi / 2$ \\
\hline 6 & $q_{6}$ & $d_{6}=0.072$ & $a_{6}=0$ & $\alpha_{6}=0$ \\
\hline
\end{tabular}

The general matrix form of robot dynamic equations without considering friction is

$$
\boldsymbol{M}(\boldsymbol{q}) \ddot{\boldsymbol{q}}+\boldsymbol{C}(\boldsymbol{q}, \dot{\boldsymbol{q}}) \dot{\boldsymbol{q}}+\boldsymbol{g}(\boldsymbol{q})=\boldsymbol{\tau}
$$

where $\mathbf{q} \in \mathbb{R}^{6 \times 1}$ is the vector of joint variables, $\mathbf{M} \in \mathbb{R}^{6 \times 6}$ is the generalized inertia matrix, $\mathbf{C} \in \mathbb{R}^{6 \times 6}$ is the Coriolis/centrifugal matrix, $\mathbf{g} \in \mathbb{R}^{6 \times 1}$ is the vector of gravity term, $\tau \in \mathbb{R}^{6 \times 1}$ is the applied torque vector. The vector/matrix parameters in (1) are calculated by the following formulas: 


$$
\begin{gathered}
\mathbf{M}=\sum_{i=1}^{6}\left(m_{i}\left(\mathbf{J}_{T_{i}}^{0}\right)^{T} \mathbf{J}_{T_{i}}^{0}+\mathbf{J}_{R_{i}}^{T} \mathbf{I}_{i} \mathbf{J}_{R_{i}}\right) \\
\mathbf{C}=\frac{1}{2}\left[\frac{\partial \mathbf{M}}{\partial \mathbf{q}}\left(\mathbf{1}_{6} \otimes \dot{\mathbf{q}}\right)+\frac{\partial \mathbf{M}}{\partial \mathbf{q}}\left(\dot{\mathbf{q}} \otimes \mathbf{1}_{6}\right)-\left(\frac{\partial \mathbf{M}}{\partial \mathbf{q}}\left(\dot{\mathbf{q}} \otimes \mathbf{1}_{6}\right)\right)^{T}\right] \\
\mathbf{g}(\mathbf{q})=\left(\frac{\partial P}{\partial \mathbf{q}}\right)^{T}
\end{gathered}
$$

where $m_{i}$ is the mass of link $i ; \mathbf{I}_{i} \in \mathbb{R}^{3 \times 3}$ is the inertia tensor of link $i$ represented in the coordinate system attached at the centroid of link $i ; \mathbf{J}_{\mathrm{R}_{i}} \in \mathbb{R}^{3 \times 6}$ and $\mathbf{J}_{T_{i}}^{0} \in \mathbb{R}^{3 \times 6}$ are rotational and translational Jacobian matrices related to frame $i$ and the base frame, respectively; $P$ is the total potential energy of robot.

\subsection{Quasi-Physical Modeling by Simscape Multibody}

Simscape Multibody provides a 3D simulation environment for multi-component mechanical systems such as robots, multibody systems, car suspension systems. Simscape Multibody gives blocks representing for links, joints, sensors and torque/force components. With a geometric structure of link, Simscape Multibody allows users to draw with simple blocks. However, you can use CAD softwares to design bodies (such as Autodesk Inventor, SolidWorks, Onshape) and MATLAB/Simscape Multibody can use these CAD files to generate the system model.

Based on the CAD models provided by ABB for robot IRB 120, we exploit Autodesk Inventor to explore the parameters of robot and construct the 3D assembly model of robot IRB 120. The approximated values of mass, link centroids, inertia tensors can be achieved by performing the physics analysis method of Autodesk Inventor as follows

$$
\text { B ase }: m_{0}=8.659
$$

$$
\begin{aligned}
\operatorname{Link} 1: m_{1} & =4.248, \mathbf{r}_{C_{1}}=\left[\begin{array}{c}
0 \\
0.054 \\
0
\end{array}\right], \\
\mathbf{I}_{1} & =\left[\begin{array}{ccc}
19.699 & 0 & 0 \\
0 & 14.484 & 0 \\
0 & 0 & 19.952
\end{array}\right] 10^{-3} \\
\operatorname{Link} 2: m_{2} & =5.412, \mathbf{r}_{C_{2}}=\left[\begin{array}{c}
-0.169 \\
0 \\
0
\end{array}\right], \\
\mathbf{I}_{2} & =\left[\begin{array}{ccc}
35.942 & 0 & 0 \\
0 & 83.522 & 0 \\
0 & 0 & 57.569
\end{array}\right] 10^{-3}
\end{aligned}
$$

$$
\begin{aligned}
\operatorname{Link} 3: m_{3} & =4.077, \mathbf{r}_{C_{3}}=\left[\begin{array}{c}
-0.012 \\
0 \\
0.023
\end{array}\right], \\
\mathbf{I}_{3} & =\left[\begin{array}{ccc}
17.562 & 0 & -1.993 \\
0 & 23.140 & 0 \\
-1.993 & 0 & 11.589
\end{array}\right] 10^{-3} \\
\operatorname{Link} 4: m_{4} & =1.832, \mathbf{r}_{C_{4}}=\left[\begin{array}{c}
0 \\
-0.007 \\
0
\end{array}\right], \\
\operatorname{Link} 5: & m_{5}=0.755, \mathbf{r}_{C_{5}}=\left[\begin{array}{c}
0 \\
0 \\
0
\end{array}\right], \\
\mathbf{I}_{4} & =\left[\begin{array}{ccc}
7.247 & 0 & 0 \\
0 & 3.919 & 0 \\
0 & 0 & 5.551
\end{array}\right] 10^{-3} \\
\mathbf{I}_{5} & =\left[\begin{array}{ccc}
1.120 & 0 & 0 \\
0 & 1.227 & 0 \\
0 & 0 & 0.559
\end{array}\right] 10^{-3} \\
\operatorname{Link} 6: m_{6} & =0.019, \mathbf{r}_{C_{6}}=\left[\begin{array}{c}
0 \\
0 \\
-0.007
\end{array}\right], \\
\mathbf{I}_{6} & =\left[\begin{array}{ccc}
2.347 & 0 & 0 \\
0 & 2.347 & 0 \\
0 & 0 & 4.123
\end{array}\right] 10^{-6}
\end{aligned}
$$

where the units of mass, length, inertia tensor are $\mathrm{kg}, \mathrm{m}$, and $\mathrm{kgm}^{2}$, respectively. Autodesk Inventor can export a robot CAD assembly file and a set of geometry files that imported into MATLAB Simscape Multibody to automatically generate the Simscape Multibody model of robot IRB 120 with the same physics description (Figure 2 and Figure 3).

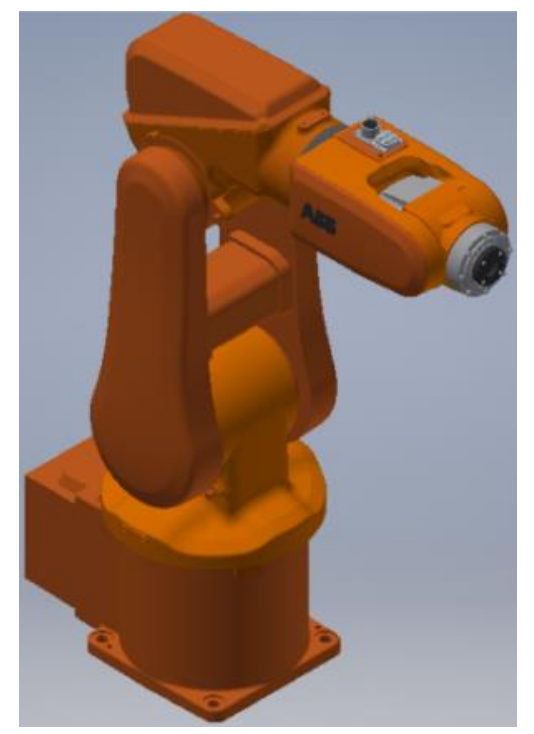

Figure 2. Autodesk Inventor 3D assembly model of robot IRB 120 


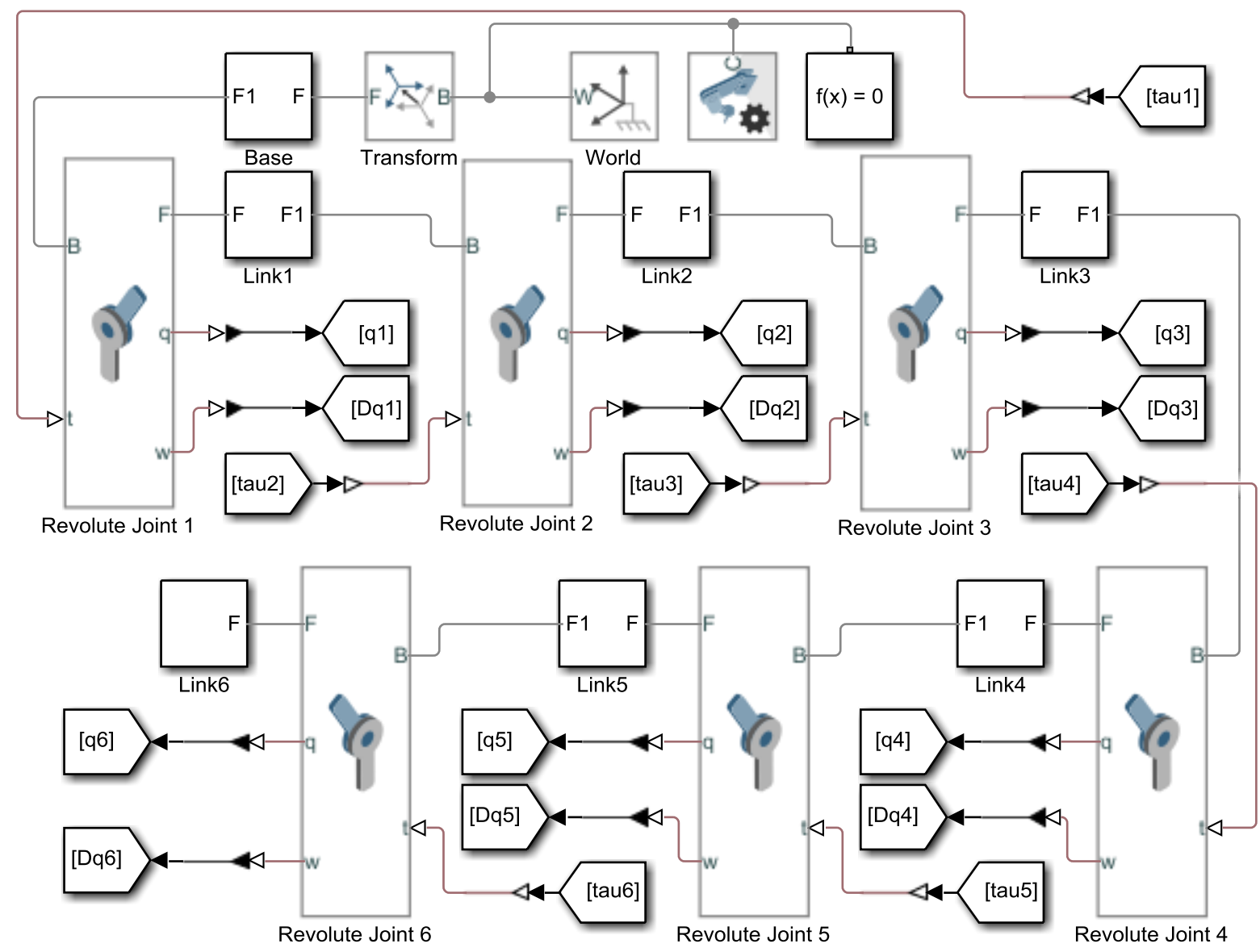

Figure 3. Autodesk Inventor $3 \mathrm{D}$ assembly model of robot IRB 120

\section{Model of Actuator Torque Degradation}

Physical constraints inherently prevent control actuators from providing unlimited torques or unlimitedly fast fluctuation [19]-[22]. In practical activities, the actuators are time-worn and may encounter many different failures. The amplitude and rate control bounds of the actuators become less and less and even less than necessary values to accomplish the tasks. These may result in inaccuracies and instability of the whole manipulator. In the followings, the type of actuator torque degradation considered in this paper, which may occur in the $i$ th actuator (Figure 4), can be defined and modeled. In Figure 4, $\tau_{d i}$ denotes the desired torque provided by the controller for actuator $i, \tau_{i}$ is the actual torque generated by the actuator $i,(i=1,2, . ., 6)$.

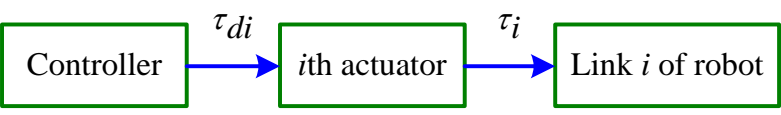

Figure 4. Block diagram of an actuator of a robot

In normal situation of actuator $i$ with its ideal inner-loop controller, $\tau_{i}$ must match $\tau_{d i}$. As stated above, a torque of a certain actuator is originally subject to a certain limit. This limit can vary depending on different sorts of actuators.

$$
\tau_{i \min } \leq \tau_{i} \leq \tau_{i \max }
$$

where $\tau_{i \min }$ and $\tau_{i \max }$ are the original minimum and maximum torque of actuator $i$, respectively. Without appropriate maintenance, actuators might degrade gradually after a period of time of operation. There are several failures which may occur such as misalignment, shaft looseness or shaft imbalance, etc. When these faults happen, the actuators will be not able to perform perfectly. One of the consequences can be a new torque limit which is tightened.

$$
\tau_{i \min }<\tau_{i \min }^{\prime} \leq \tau_{i} \leq \tau_{i \max }^{\prime}<\tau_{i \max }
$$

where $\tau_{i \text { min }}^{\prime}$ and $\tau_{i \max }^{\prime}$ are the new minimum and maximum torque of defected actuator $i$. This is the first kind of actuator torque degradation, namely Boundary Degradation of Torque (BDT).

Actuator problem can be classified not only in BDT failure but also in Boundary Degradation of Torque Rate (BDTR) failure - the second kind of torque degradation. Due to physical constraints, an actuator torque is not able to fluctuate at unbounded speed. The increasing and decreasing rates of torque are limited by constant values 
described as:

$$
\left|\frac{d \tau_{i}}{d t}\right| \leq \delta_{i}
$$

where $\delta_{i}$ is the original torque rate limit of actuator $i$. Robot manufacturers have to concern about those values when choosing the most suitable motors for different parts of a robot. Those values may decline as follows after working for a long time due to several causes.

$$
\left|\frac{d \tau_{i}}{d t}\right| \leq \delta_{i}^{\prime}<\delta_{i}
$$

where $\delta_{i}^{\prime}$ is the actual torque rate limit of defected actuator $i$. A low rate leads to delay in generating an appropriate torque. This failure prevents the actuator from reacting quickly enough so that the end-effector may not track the desired trajectory.

In fact, actuators face another fault. The third kind of torque degradation is Proportional Degradation of Torque (PDT). The torque generated by a defected motor loses a certain proportion of its effectiveness.

$$
\tau_{i}=k_{i} \cdot \tau_{d i}
$$

where $k_{i}$ is the proportion of torque loss of defected actuator $i,\left(0<k_{i}<1\right)$. Note that actuators operate in failure-free case if $k_{i}=1$.

\section{Backstepping Control of Robot IRB 120}

To facilitate the design of the backstepping controller of robot IRB 120 in state-space representations, we define $\mathbf{x}_{1}=\mathbf{q}, \mathbf{x}_{2}=\dot{\mathbf{x}}_{1}=\dot{\mathbf{q}}$. From (1), we have

$$
\dot{\mathbf{x}}_{2}=\mathbf{M}^{-1}\left(\boldsymbol{\tau}-\mathbf{C} \mathbf{x}_{2}-\mathbf{g}\right)
$$

Taking $\mathbf{q}_{d} \in \mathbb{R}^{6 \times 1}$ is the desired trajectory vector. The detailed design of backstepping controller is as follows.

Step 1: Firstly, consider the following signal vectors:

$$
\begin{aligned}
& \mathbf{z}_{1}=\mathbf{x}_{1}-\mathbf{q}_{d} \\
& \mathbf{x}_{2 d}=-\mathbf{c}_{1} \mathbf{z}_{1}+\dot{\mathbf{q}}_{d} \\
& \mathbf{z}_{2}=\mathbf{x}_{2}-\mathbf{x}_{2 d}
\end{aligned}
$$

where $\mathbf{z}_{1} \in \mathbb{R}^{6 \times 1}$ is the error signal vector of joint variables, $\quad \mathbf{x}_{2 d} \in \mathbb{R}^{6 \times 1}$ is the virtual variable vector, $\mathbf{z}_{2} \in \mathbb{R}^{6 \times 1}$ is the error signal vector, $\mathbf{c}_{1} \in \mathbb{R}^{6 \times 6}$ is the diagonal matrix in which all its main diagonal entries are positive constants. Thus:

$$
\dot{\mathbf{z}}_{1}=\dot{\mathbf{x}}_{1}-\dot{\mathbf{q}}_{d}=\mathbf{x}_{2}-\dot{\mathbf{q}}_{d}=\mathbf{z}_{2}+\mathbf{x}_{2 d}-\dot{\mathbf{q}}_{d}=\mathbf{z}_{2}-\mathbf{c}_{1} \mathbf{z}_{1}
$$

The first Lyapunov function to design the backstepping controller is defined as

$$
V_{1}=\frac{1}{2} \mathbf{z}_{1}^{T} \mathbf{z}_{1}
$$

The derivative of $V_{1}$ is obtained as

$$
\dot{V}_{1}=\mathbf{z}_{1}^{T} \dot{\mathbf{z}}_{1}=\mathbf{z}_{1}^{T}\left(\mathbf{z}_{2}-\mathbf{c}_{1} \mathbf{z}_{1}\right)=-\mathbf{z}_{1}^{T} \mathbf{c}_{1} \mathbf{z}_{1}+\mathbf{z}_{1}^{T} \mathbf{z}_{2}
$$

Absolutely, $\dot{V}_{1} \leq 0$ when all elements of $\mathbf{z}_{2}$ are all zero. Therefore, it is necessary to make $\mathbf{z}_{2}$ convergent.

Step 2: From (10), (12), and (13), we have

$$
\dot{\mathbf{z}}_{2}=\dot{\mathbf{x}}_{2}-\dot{\mathbf{x}}_{2 d}=\mathbf{M}^{-1}\left(\boldsymbol{\tau}-\mathbf{C} \mathbf{x}_{2}-\mathbf{g}\right)+\mathbf{c}_{1} \dot{\mathbf{z}}_{1}-\ddot{\mathbf{q}}_{d}
$$

Consider the second Lyapunov function given by

$$
V_{2}=V_{1}+\frac{1}{2} \mathbf{z}_{2}^{T} \mathbf{z}_{2}
$$

The derivative of $V_{2}$ is obtained as

$$
\dot{V}_{2}=\dot{V}_{1}+\mathbf{z}_{2}^{T} \dot{\mathbf{z}}_{2}
$$

Applying (16) and (17) to (19) has

$$
\begin{aligned}
\dot{V}_{2}= & -\mathbf{z}_{1}^{T} \mathbf{c}_{1} \mathbf{z}_{1}+\mathbf{z}_{1}^{T} \mathbf{z}_{2} \\
& +\mathbf{z}_{2}^{T}\left[\mathbf{M}^{-1}\left(\boldsymbol{\tau}-\mathbf{C} \mathbf{x}_{2}-\mathbf{g}\right)+\mathbf{c}_{1} \dot{\mathbf{z}}_{1}-\ddot{\mathbf{q}}_{d}\right]
\end{aligned}
$$

Besides, we have

$$
\mathbf{z}_{1}^{T} \mathbf{z}_{2}=\mathbf{z}_{2}^{T} \mathbf{z}_{1}
$$

Substituting (21) into (20) gives

$$
\begin{aligned}
\dot{V}_{2}= & -\mathbf{z}_{1}^{T} \mathbf{c}_{1} \mathbf{z}_{1}+\mathbf{z}_{2}^{T} \mathbf{z}_{1} \\
& +\mathbf{z}_{2}^{T}\left[\mathbf{M}^{-1}\left(\boldsymbol{\tau}-\mathbf{C x}_{2}-\mathbf{g}\right)+\mathbf{c}_{1} \dot{\mathbf{z}}_{1}-\ddot{\mathbf{q}}_{d}\right]
\end{aligned}
$$

To achieve the control objective, the control torque is designed as

$$
\boldsymbol{\tau}=\mathbf{C} \mathbf{x}_{2}+\mathbf{g}+\mathbf{M}\left(-\mathbf{c}_{1} \dot{\mathbf{z}}_{1}+\ddot{\mathbf{q}}_{d}-\mathbf{z}_{1}-\mathbf{c}_{2} \mathbf{z}_{2}\right)
$$

where $\mathbf{c}_{2} \in \mathbb{R}^{6 \times 6}$ is the diagonal matrix in which all its main diagonal entries are positive constants. By the control torque introduced above, substituting (23) into (22) yields

$$
\dot{V}_{2}=-\mathbf{z}_{1}^{T} \mathbf{c}_{1} \mathbf{z}_{1}-\mathbf{z}_{2}^{T} \mathbf{c}_{2} \mathbf{z}_{2} \leq 0
$$

Equation (24) indicates that $V_{2}$ is convergent. Hence, $\mathbf{z}_{1}$ and $\mathbf{z}_{2}$ are convergent. We can conclude that, $t \rightarrow \infty$, $\mathbf{x}_{1} \rightarrow \mathbf{q}_{d}$, and the system is stable with control input (23). It can be noted that the proposed backstepping control heavily depends on system information especially the ability to deliver required torque of the robot actuators.

\section{Simulation Results and Discussions}

In this section, the effect of the three faults on robot IRB 
120 (controlled by backstepping methodology) is verified by numerical simulations. In the base Cartesian space, the entire desired trajectory lying on the plane perpendicular to $y_{0}$-axis and having $y$-component coordinate $-0.3 \mathrm{~m}$ is defined as a half of a circle whose radius is $0.1 \mathrm{~m}$ (Figure 5). The center of the circle in the base coordinate system is $[0,-0.3,0.5]^{T}$. The initial position and the end point are $[0,-0.3,0.6]^{T}$ and $[0,-0.3,0.4]^{T}$, respectively.

In order to evaluate the effect of the failures, the system is simulated in the different cases. Every jo int of the 6-DOF robot is subject to each of the three failures separately and independently. Thus, 18 different cases are simulated. In each case, the percentages of actuator torque losses are considered depending on each joint so that the effect of failures can be expressed clearly. As a certain joint of the robot is under a failure, it is obvious that all other jo ints are also somewhat affected. However, the changes in the torques of the remain ing jo ints are not as re markable as that of the faulty joint. Therefore, only the torque generated by the actuator which is experiencing the failures is shown. Of course, the effect on the End-Effector trajectory is also displayed through its projections on the three base $2 \mathrm{D}$

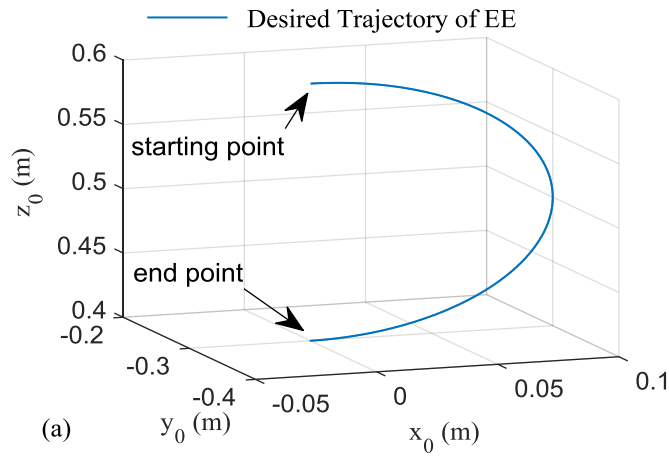

Cartesian planes. In simulations, BDT failure is active at 0.4 seconds after End-Effector has followed the desired half of the circle. BDTR and PDT failures can be active at the beginning of the simu lations. The results are as follows.

\subsection{Boundary Degradation of Torque Failure}

In Figure 6, BDT failure causes tracking errors proportional to the degradation loss. The situation become worse considering the failure is in joint 2 or jo int 3 . In Figure 7 and Figure 8, serious tracking errors due to BDT failures happen; small BDT failure causes large tracking errors. The robot cannot follow half of the circle trajectory. Figure 9 ind icates that tracking error is small and does not affect the trajectory of the robot even the torque loss level is at $50 \%$. In Figure 10, the robot seems to provide good tracking results but does not follow the reference at the end of the trajectory. In Figure 11, s mall tracking errors can be spotted, however the errors do not affect the trajectory of the robot much. It is obvious since joint 6 with the end-effector does not need large control torque.

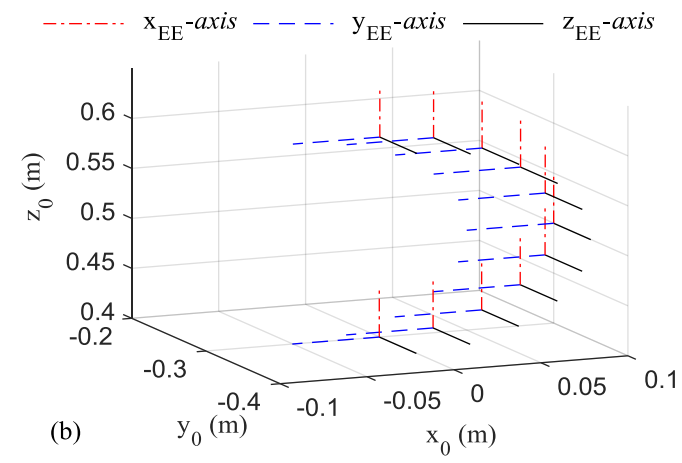

Figure 5. (a) Desired Trajectory and (b) Orientation of End-Effector (EE) in the base Cartesian space
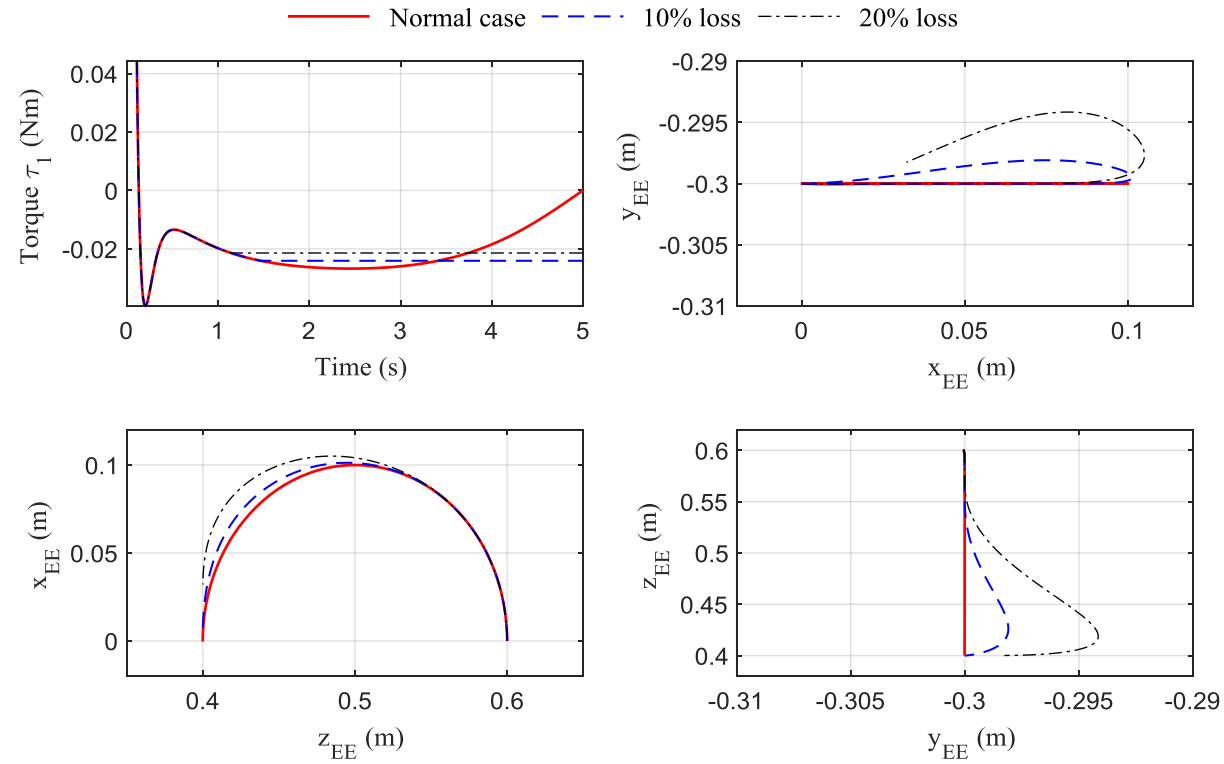

Figure 6. Effect of BDT failure of joint 1 on End-Effector trajectory projected on three Cartesian planes 

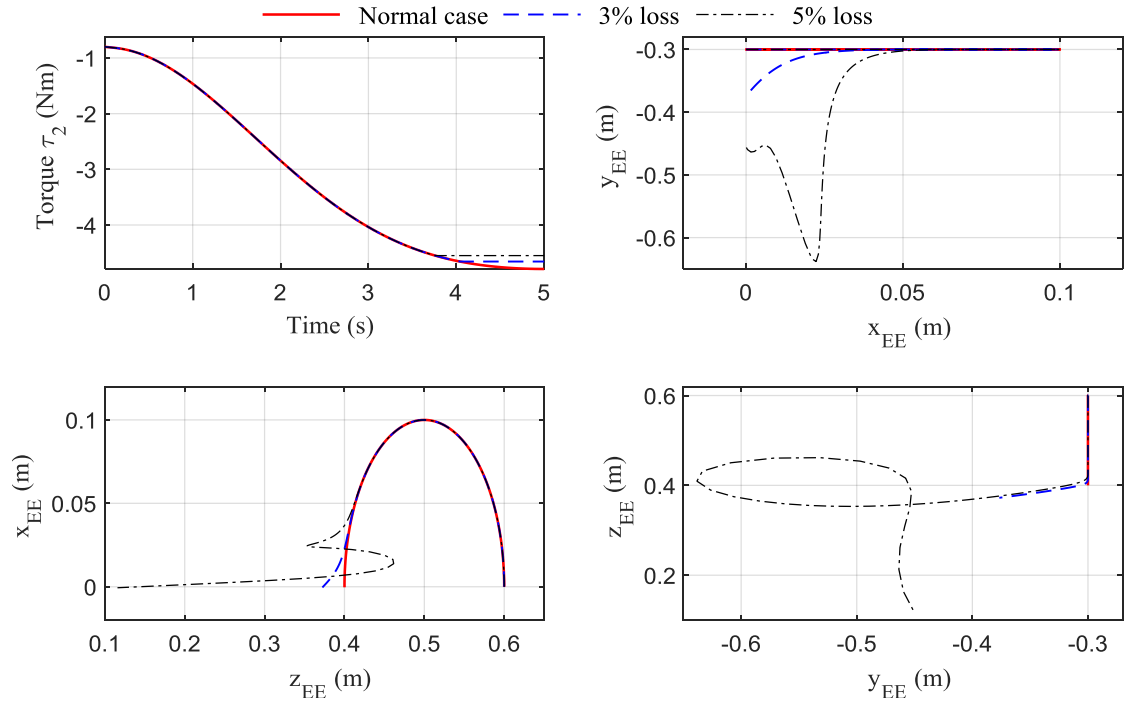

Figure 7. Effect of BDT failure of joint 2 on End-Effector trajectory projected on three Cartesian planes
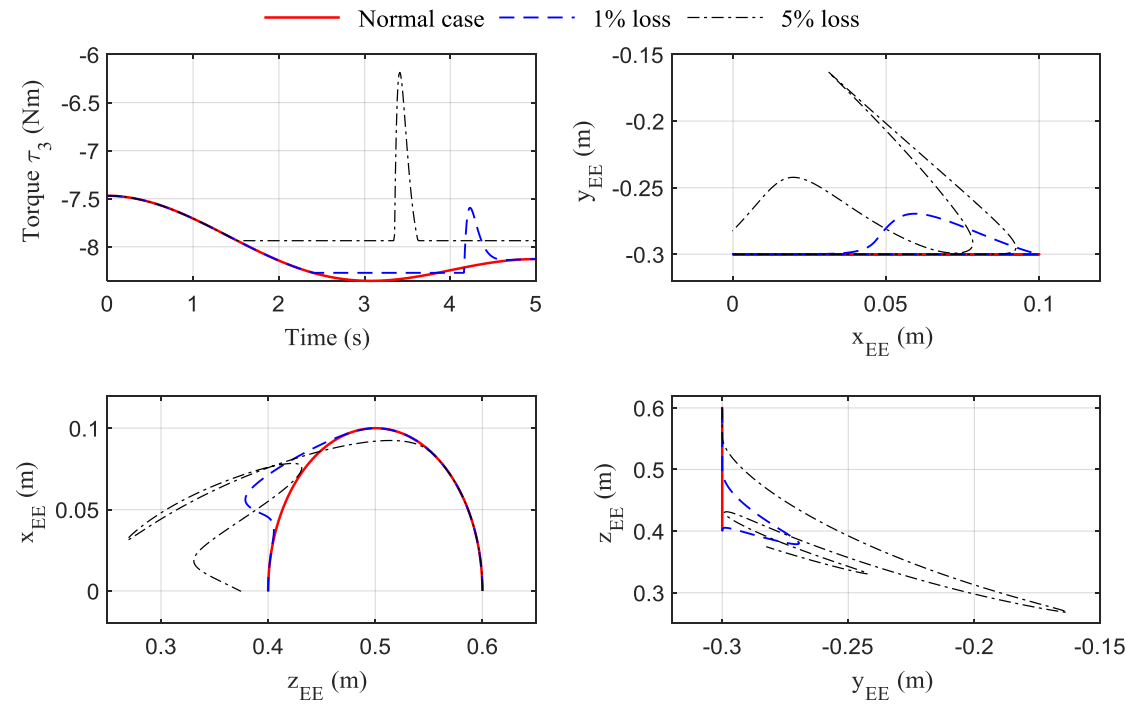

Figure 8. Effect of BDT failure of joint 3 on End-Effector trajectory projected on three Cartesian planes
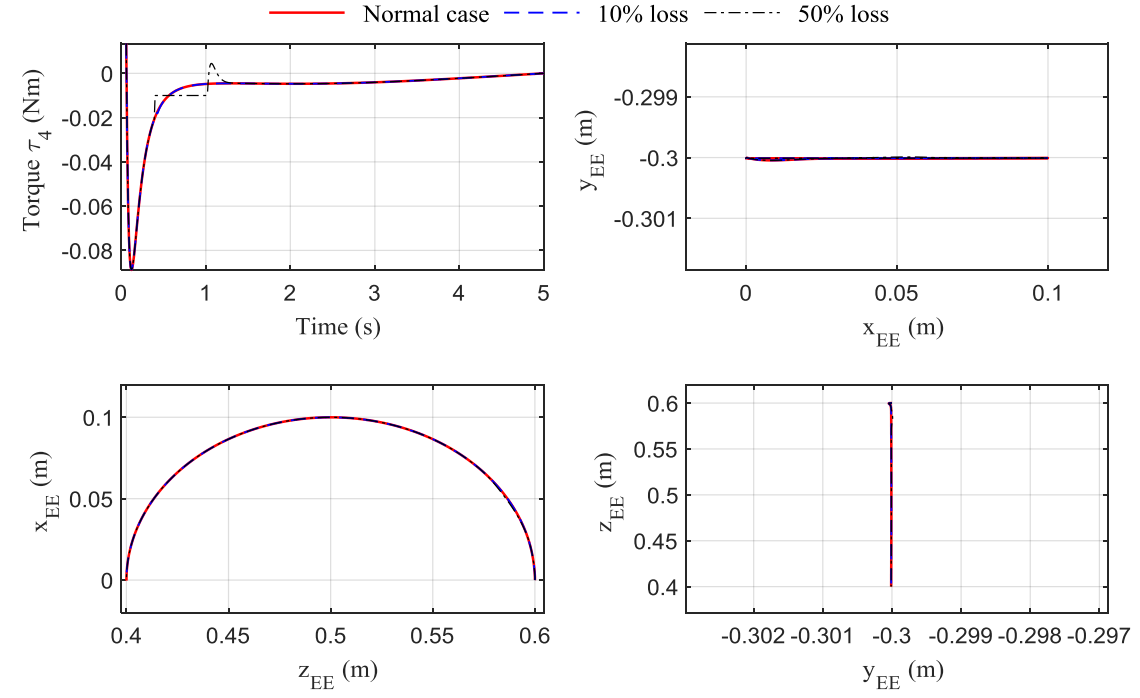

Figure 9. Effect of BDT failure of joint 4 on End-Effector trajectory projected on three Cartesian planes 

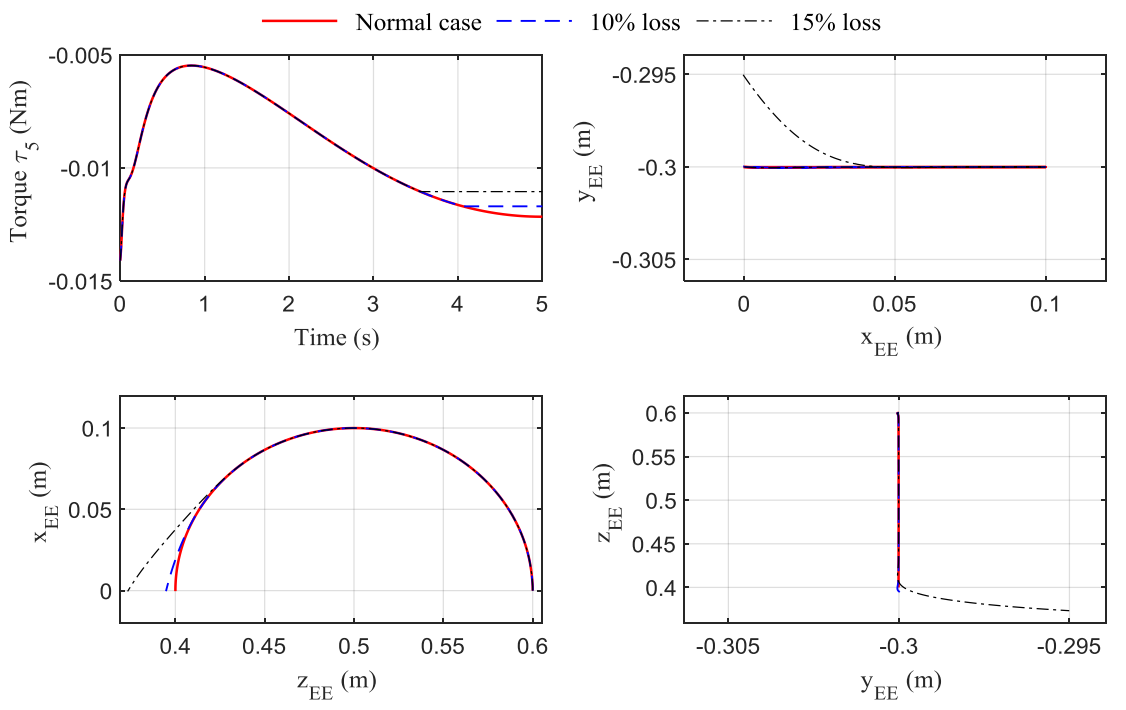

Figure 10. Effect of BDT failure of joint 5 on End-Effector trajectory projected on three Cartesian planes
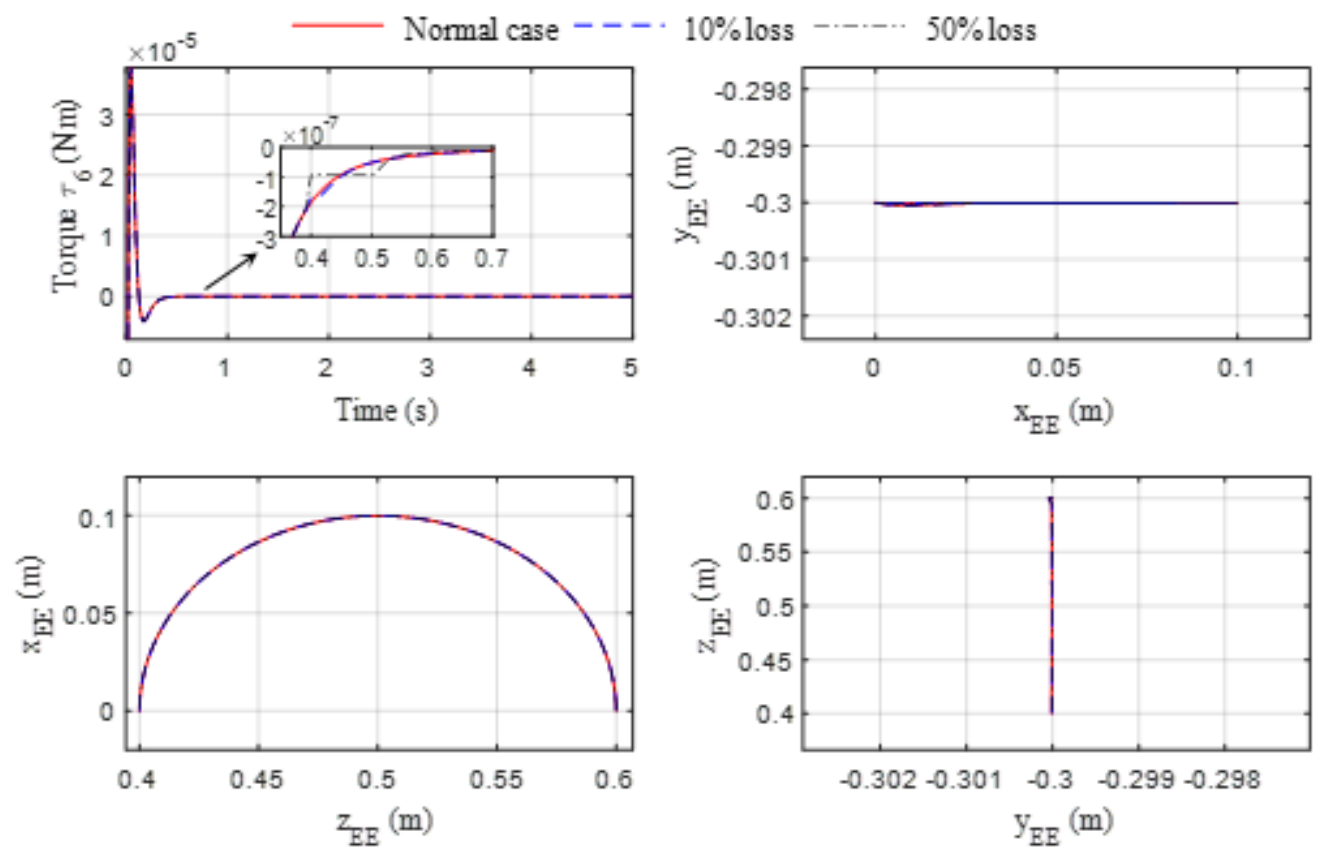

Figure 11. Effect of BDT failure of joint 6 on End-Effector trajectory projected on three Cartesian planes

\subsection{Boundary Degradation of Torque Rate Failure}

In Figure 12, BDTR failure does not affect the trajectory of the robot when happening at joint 1 . In Figure 13, BDTR failure causes serious errors. In $4 \%$ loss case, the tracking performance is guaranteed, however increasing loss level to $4.5 \%$ cause considerable errors indicat ing that the joint is very sensitive to BDTR. The results indicate the joint 2 having high sensitivity to BDTR. In Figure 14, and Figure 15 , BDTR failure at $10.5 \%$ level does not affect the trajectory of the robot when occurs in joint 4 but creates visible errors if implemented in joint 3. In Figure 16 and 17, BDTR failure causes a small tracking errors, the robot is close to the desired trajectory. In Figure 16, the impact torque has a slight vibration when starting the rotation process, meanwhile in Figure 17; torque has a strong tug, which may lead to harmful vibration to actuators. Torque rate degradation does not have serious effects on joint 4,5 , and 6 due to lighter masses compared to joints 1,2 , and 3 . The end-effector still tracks the reference trajectory at torque loss of $50 \%$. 

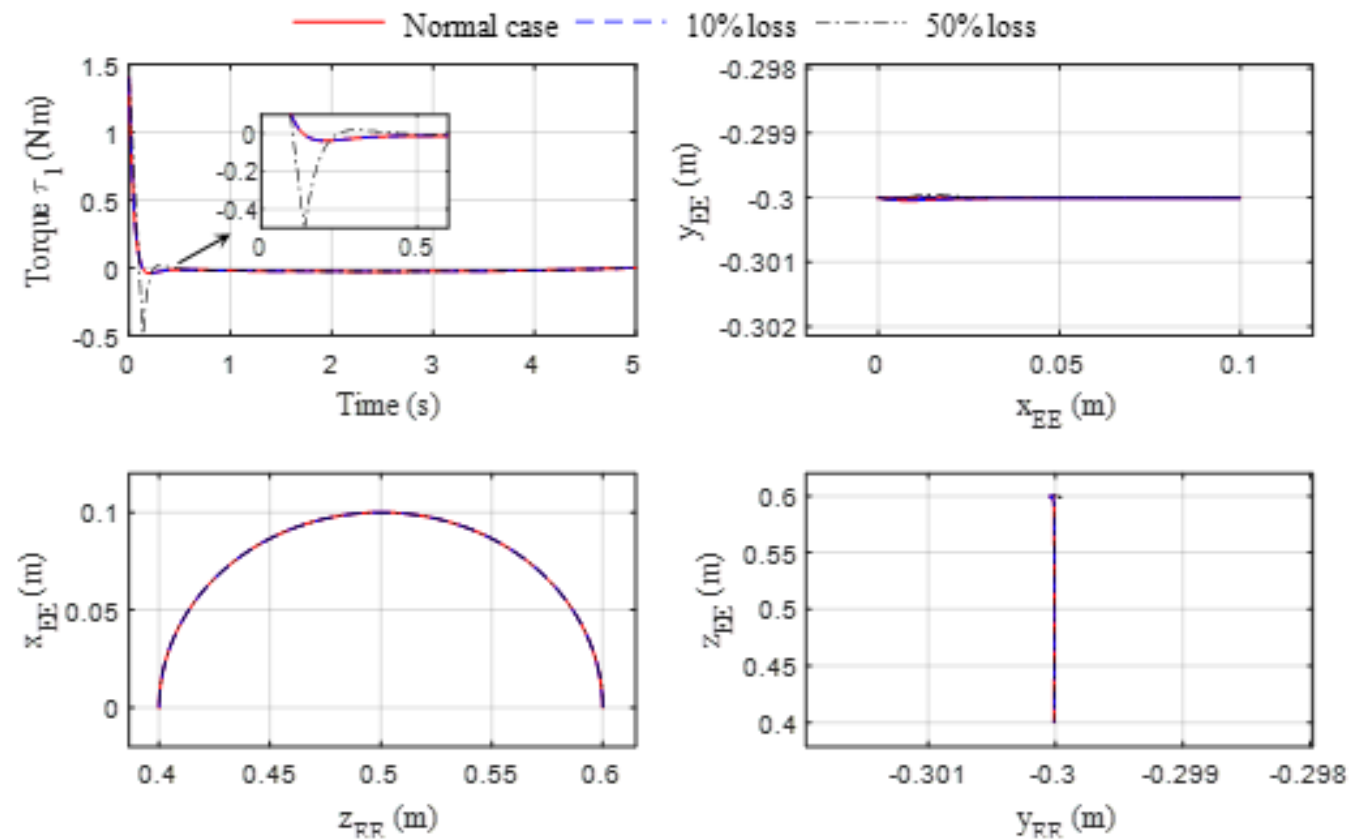

Figure 12. Effect of BDTR failure of joint 1 on End-Effector trajectory projected on three Cartesian planes Normal case $--\cdots \cdot 4 \%$ loss $-\cdot \cdot \cdot \cdot 4.5 \%$ loss
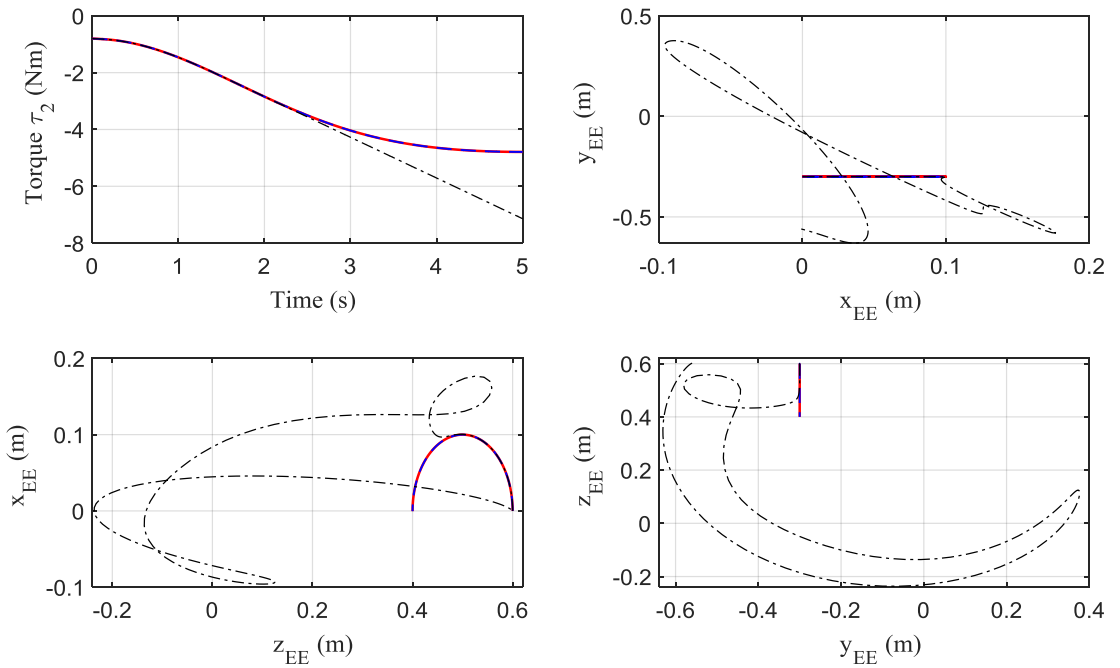

Figure 13. Effect of BDTR failure of joint 2 on End-Effector trajectory projected on three Cartesian planes 

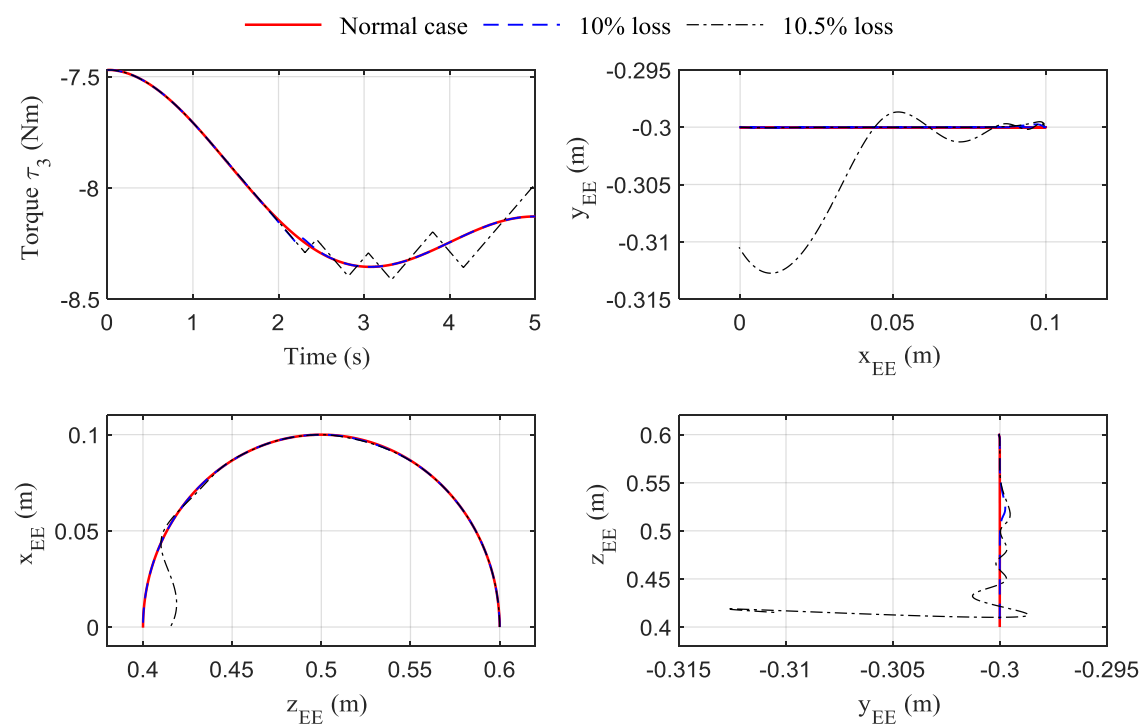

Figure 14. Effect of BDTR failure of joint 3 on End-Effector trajectory projected on three Cartesian planes

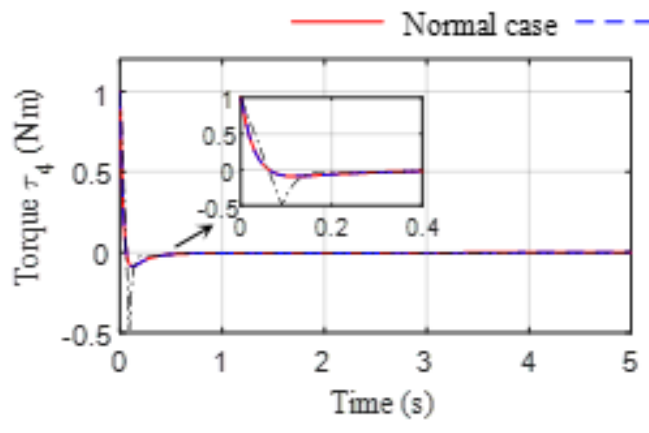

$10 \%$ loss -
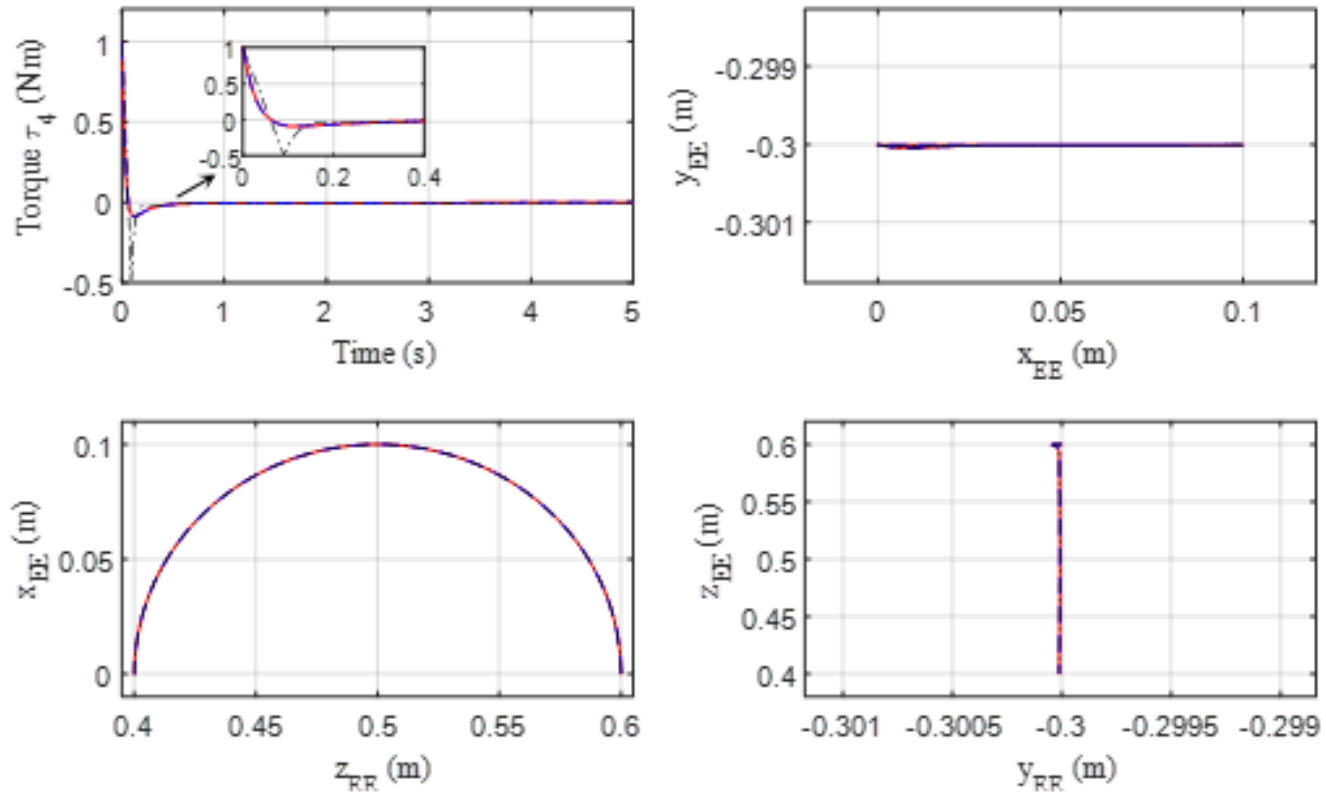

Figure 15. Effect of BDTR failure of joint 4 on End-Effector trajectory projected on three Cartesian planes 

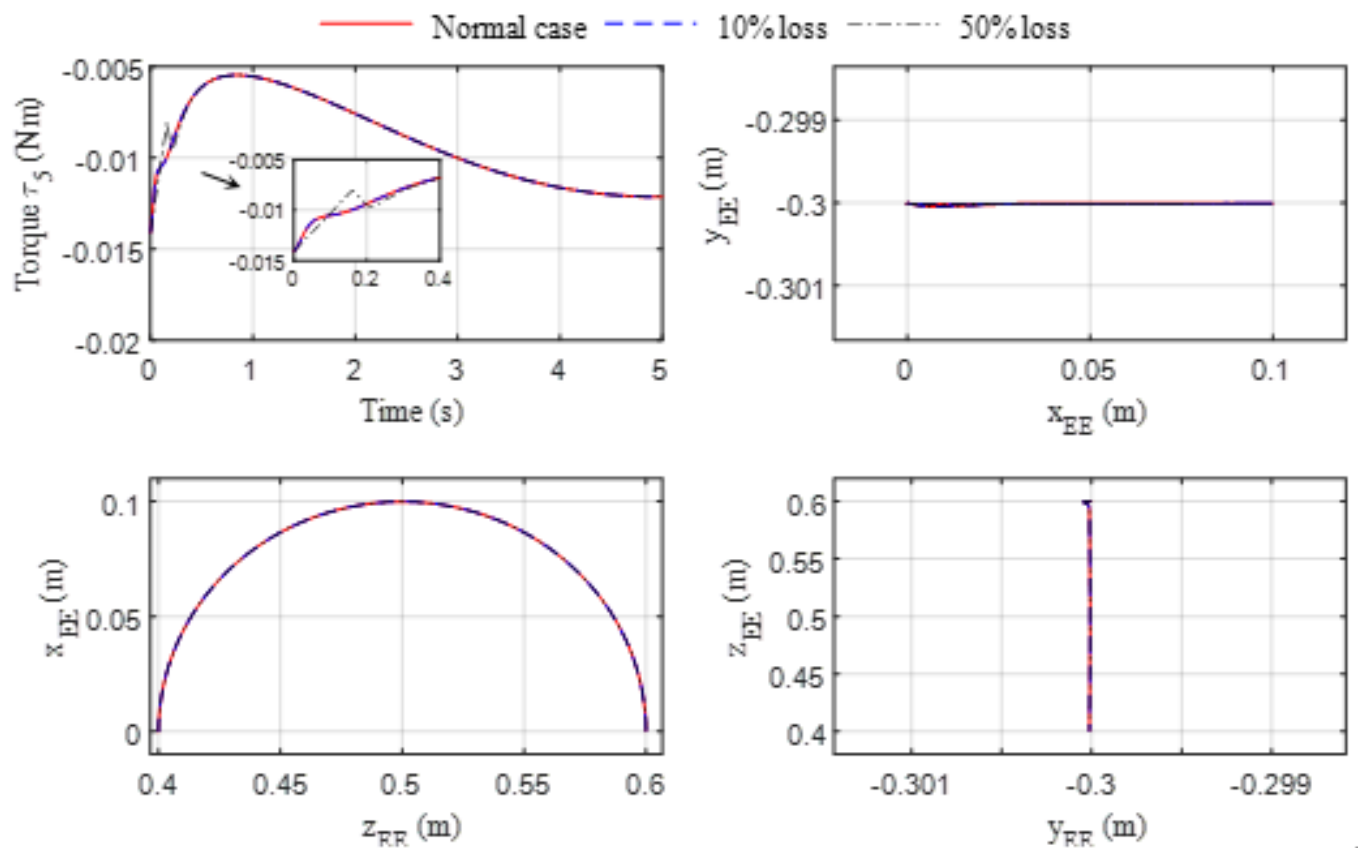

Figure 16. Effect of BDTR failure of joint 5 on End-Effector trajectory projected on three Cartesian planes
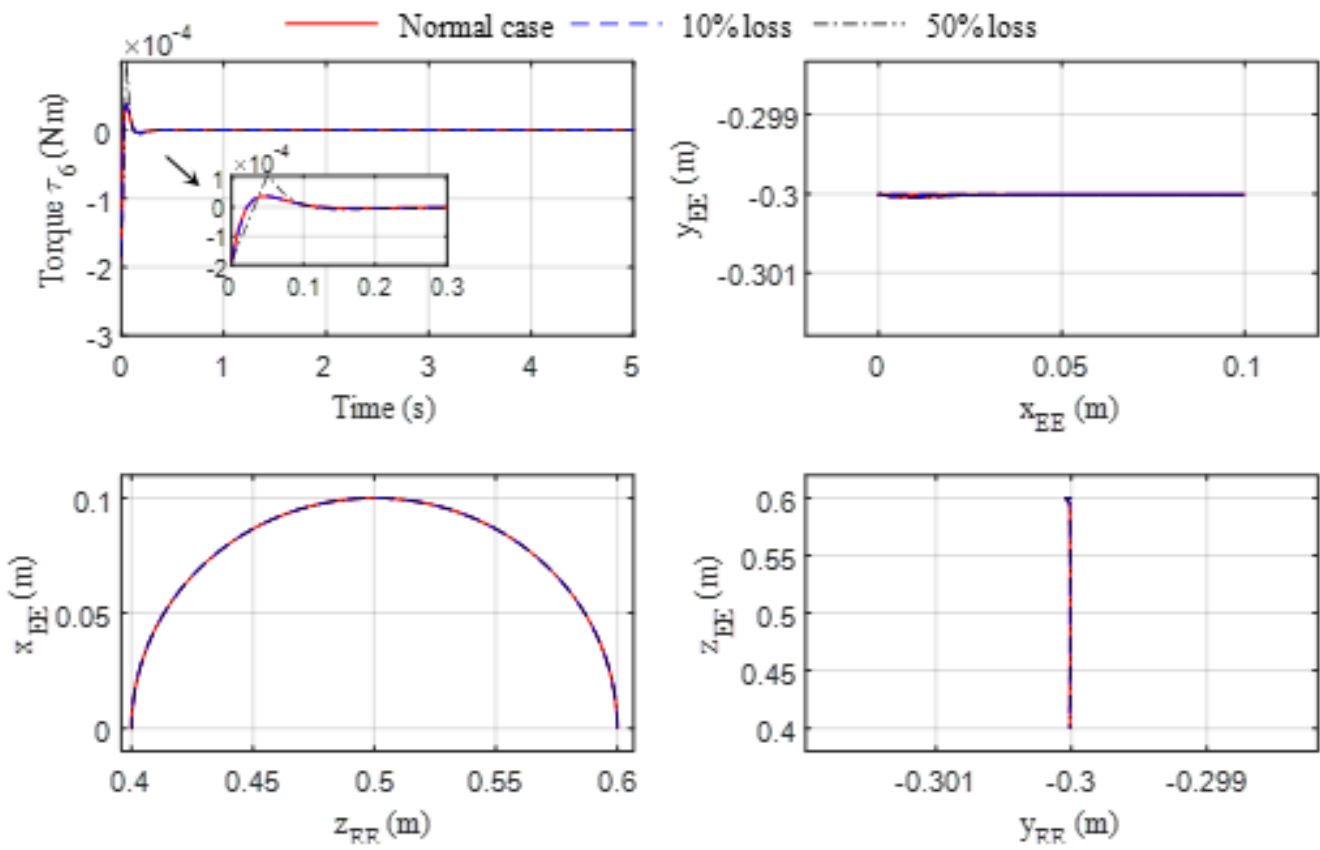

Figure 17. Effect of BDTR failure of joint 6 on End-Effector trajectory projected on three Cartesian planes

\subsection{Proportional Degradation of Torque Failure}

Figure 18 to 21 show the system performance against PDT failure for jo in 1 to 4 , respectively. It clearly indicates that the failure has a relatively large influence on joint 2 and 3. The effect is not visible in join 1 and 4. From Figure 22 to Figure 23, PDT failu re causes a small tracking errors, robot is close to the desired trajectory. In general, it can be seen that the effect of the BDTR failure is the most serious while that of PDT is the most tolerable. Additionally, failures in joint 2 and 3 result in impressive impacts on the joints that due to the inherent structure of the manipulator. Initially, all the joints require a massive torque to operate. However, owing to the robot structure, the necessary torques of joint 1,2 , and 3 are bigger than those of jo int 4,5 , and 6 . This conclusion can be drawn for all three kinds of actuator failures. However, the loss percentage has to reach a considerably high value to affect the tracking performance. 

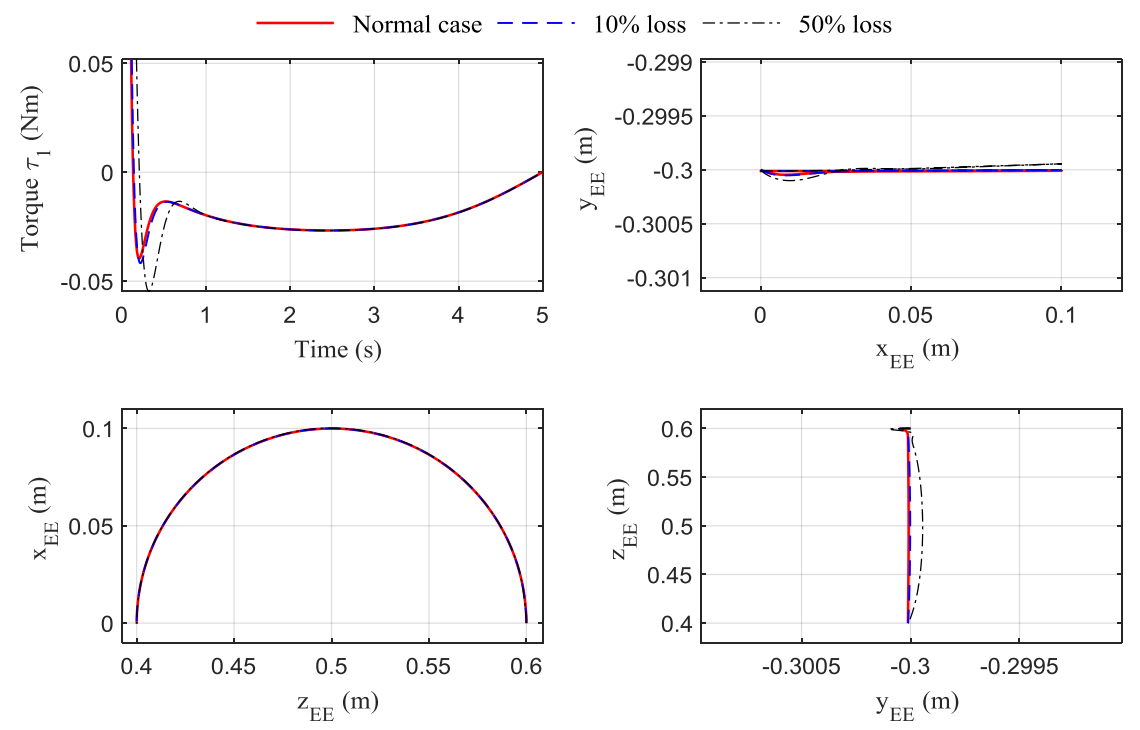

Figure 18. Effect of PDT failure of joint 1 on End-Effector trajectory projected on three Cartesian planes
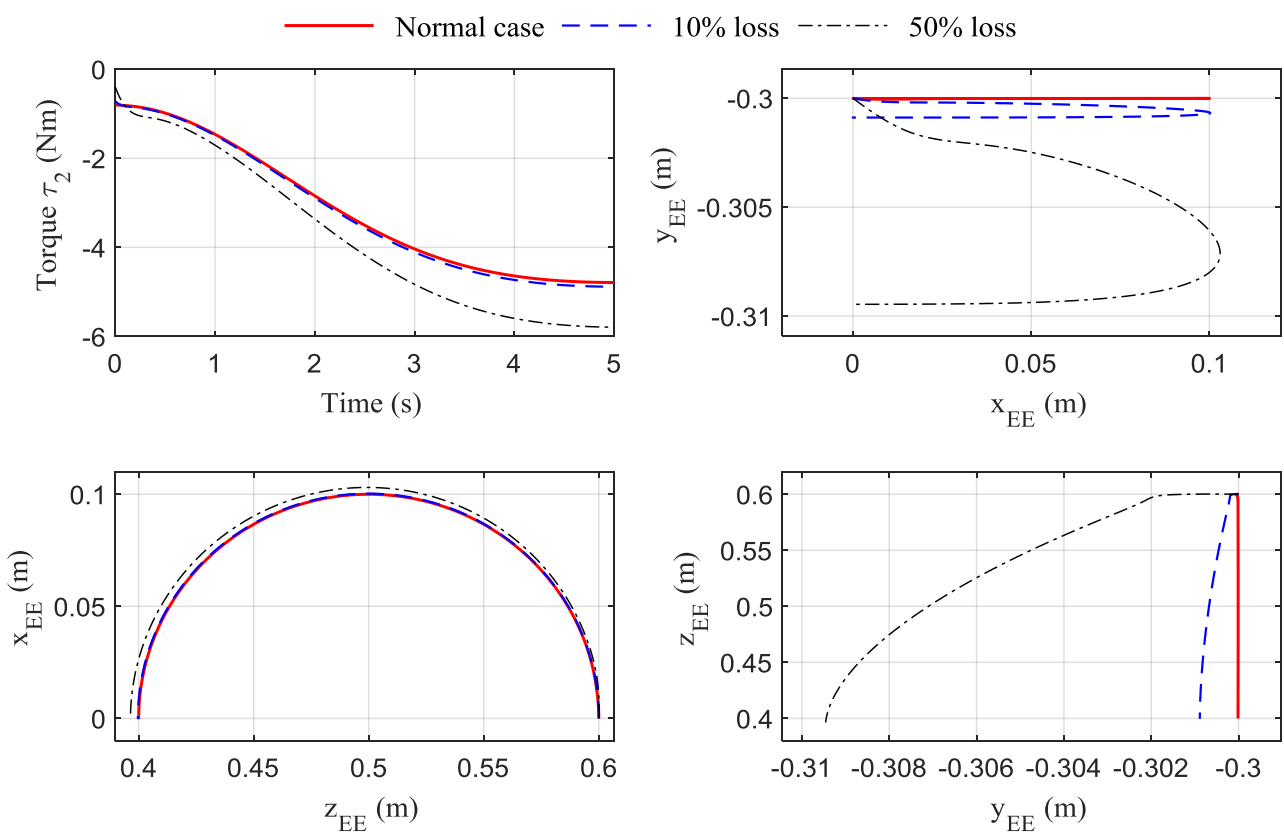

Figure 19. Effect of PDT failure of joint 2 on End-Effector trajectory projected on three Cartesian planes 

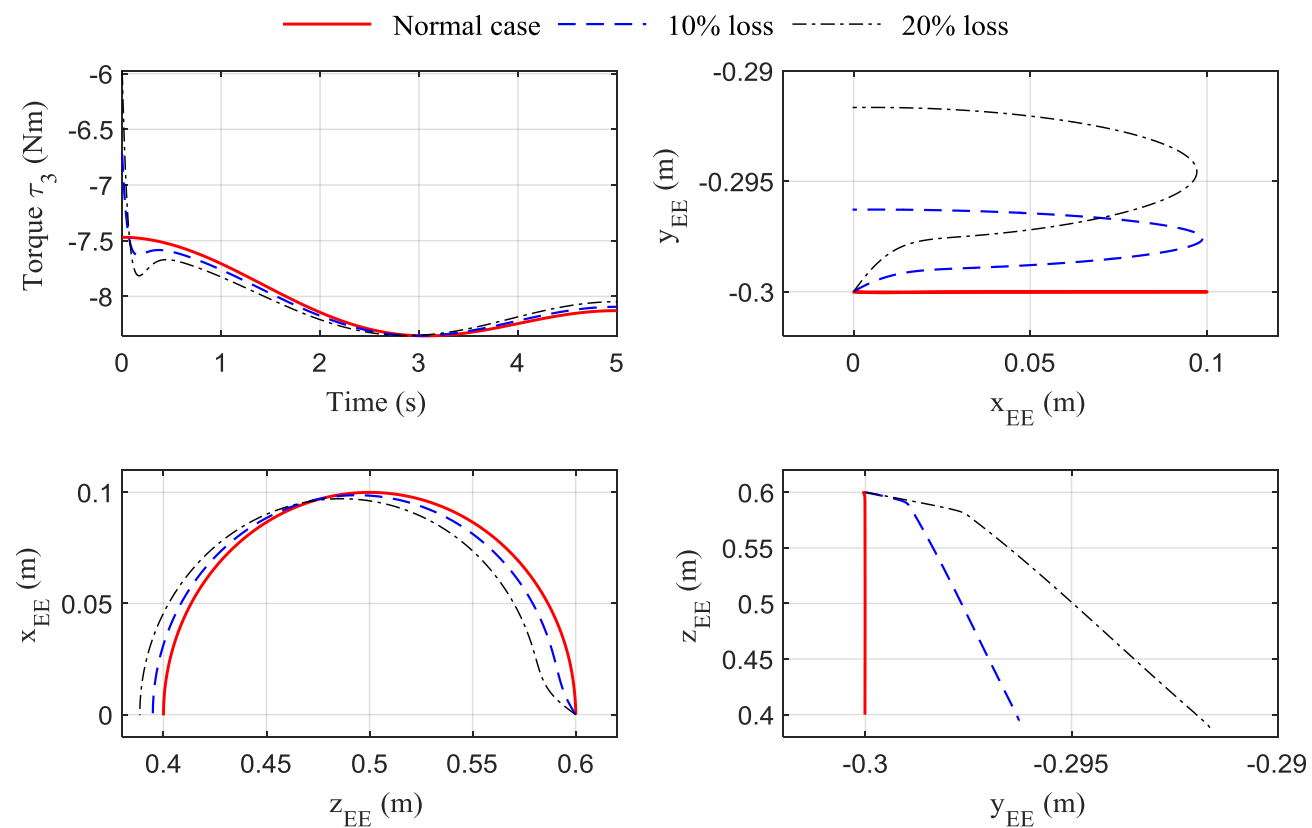

Figure 20. Effect of PDT failure of joint 3 on End-Effector trajectory projected on three Cartesian planes
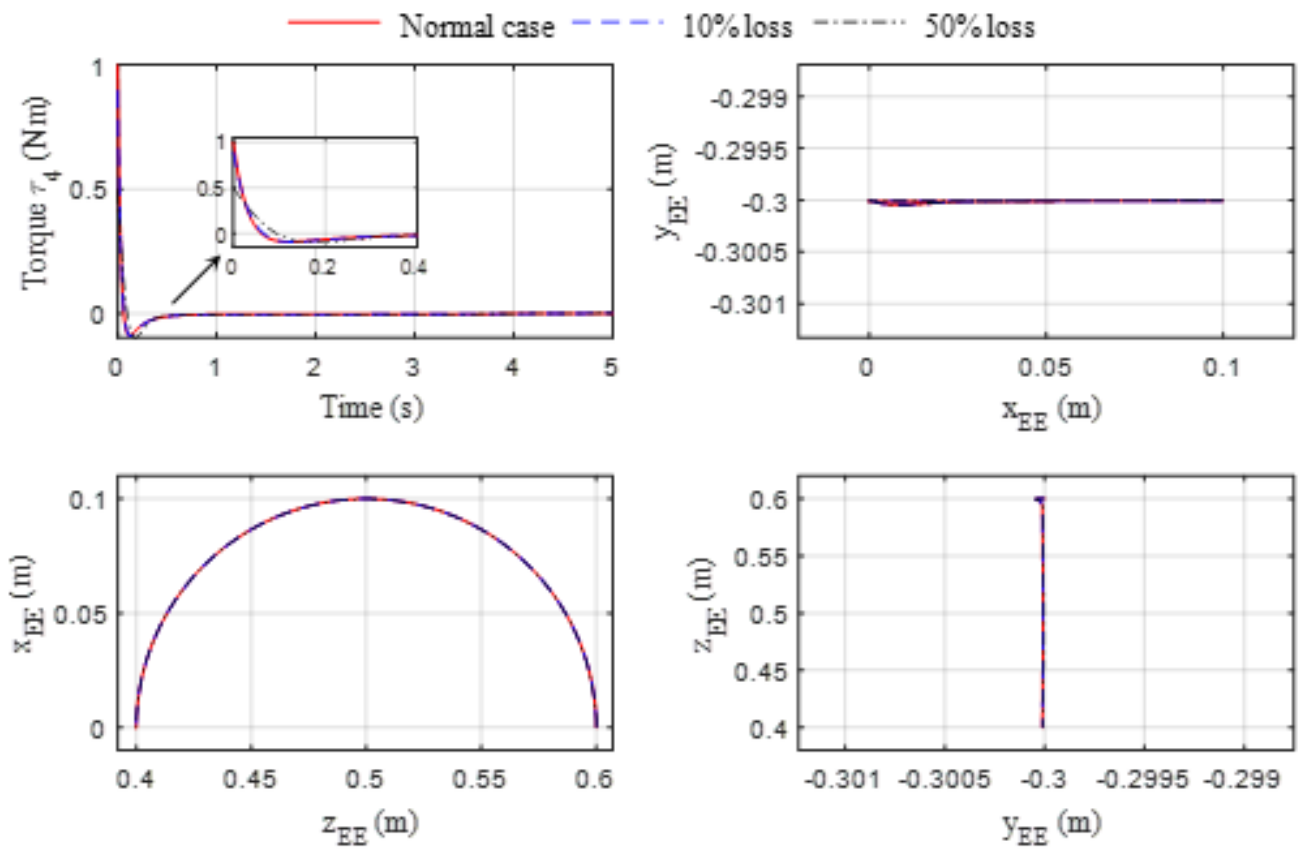

Figure 21. Effect of PDT failure of joint 4 on End-Effector trajectory projected on three Cartesian planes 

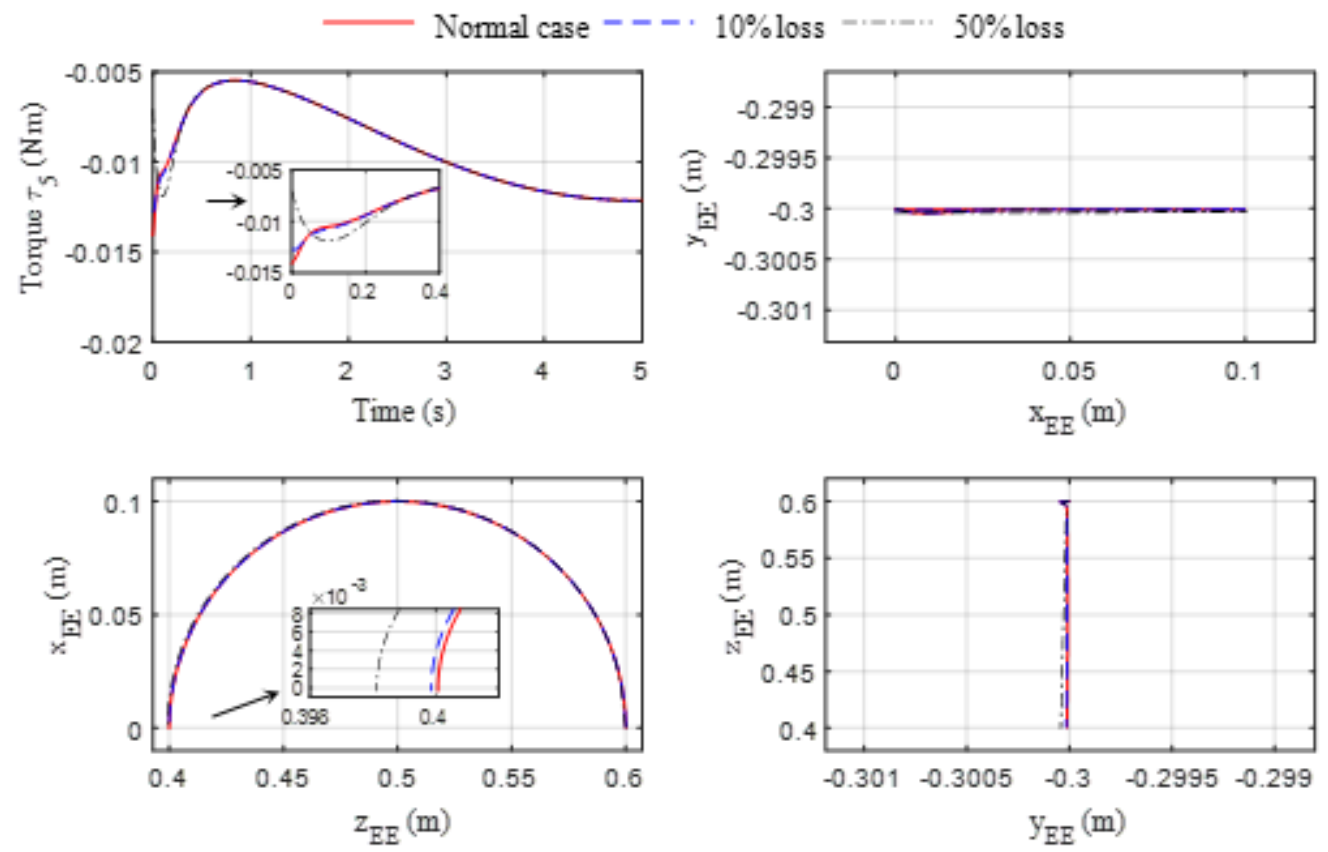

Figure 22. Effect of PDT failure of joint 5 on End-Effector trajectory projected on three Cartesian planes
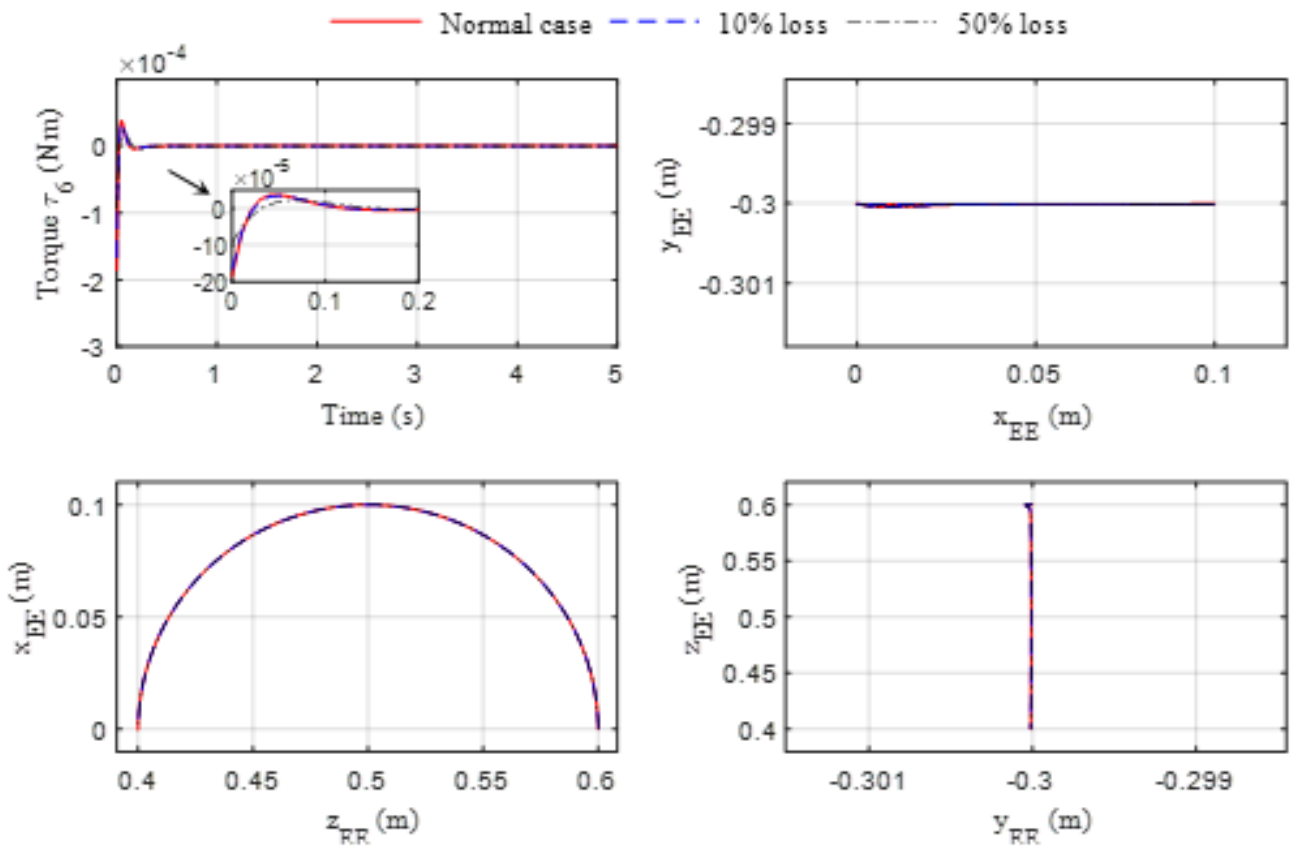

Figure 23. Effect of PDT failure of joint 6 on End-Effector trajectory projected on three Cartesian planes

\section{Conclusions}

In this study, three cases of actuator torque degradation faults are defined and presented namely: Boundary Degradation of Torque, Boundary Degradation of Torque Rate and Proportional Degradation of Torque. In order to judge the control ability of backstepping controller which is applied to the robot IRB 120 suffering from each of above failures, several simu lation scenarios are conducted. Thanks to Simscape Multibody, the quasi-physical modeling of the robot has appreciable accuracy and reliability. The simulation results provided indicate the considerable effect of actuator torque degradation faults on the performance of a 6-DOF manipulator. The controller which is specifically designed in normal situation does not meet the control requirements when the robot is subject to actuator failures in torque degradation. It can be observed from the simulations that there remain some interesting yet challenging open problems for this study. As a future work, some efficient control strategies need to be designed somehow to guarantee the manipulator efficiency and accuracy. 


\section{Acknowledgements}

This research is supported by Hanoi University of Science and Technology research grant T2018-PC-053.

\section{REFERENCES}

[1] B. Siciliano and O. Khatib, Eds., Springer Handbook of Robotics, Springer International Publishing, Cham, Zug, Switzerland, 2016.

[2] A. A. Awelewa, K. C. Mbanisi, S. O. Majekodunmi, I. A. Odigwe, A. F. Agbetuyi, and I. A. Samuel. Development of a Prototype Robot Manipulator for Industrial Pick-and-Place Operations, International Journal of Mechanical \& Mechatronics Engineering, Vol.13, No.05, 20-28, 2013.

[3] S. Yaghoubi. Robotics and Automations in Construction: Advanced Construction and Future Technology, International Journal of En gineering \& Technology, Vol.13, No.03, 19-25, 2013.

[4] R. Alizade, "Structural Synthesis of Robot Manipulators by Using Screw with Variable Pitch," Universal Journal of Mechanical Engineering, Vol.7, No.2, 50-63, 2019.

[5] H. Abdi, S. Nahavandi, Y. Fray man, and A. A. Maciejewski. Optimal mapping of joint faults into healthy joint velocity space for fault-tolerant redundant manipulators, Robotica, Vol.30, No.4, 635-648, 2012.

[6] C. L. Lewis and A. A. Maciejewski. Fault tolerant operation of kinematically redundant manipulators for locked joint failures, IEEE Transactions on Robotics and Automation, Vol.13, No.4, 622-629, 1997.

[7] J. D. English and A. A. Maciejewski. Fault tolerance for kinematically redundant manipulators: Anticipating free-swinging joint failures, IEEE Transactions on Robotics and Automation, Vol.14, No.4, 566-575, 1998.

[8] Y. She, W. Xu, Z. Wang, and J. Peng. Inverse kinematics of SSRMS-type manipulators with single joint locked failure, 2013 IEEE International Conference on Robotics and Biomimetics (ROBIO), 7-12, 2013.

[9] J. D. English and A. A. Maciejewski. Failure Tolerance through Active Braking: A Kinematic Approach, The International Journal of Robotics Research, Vol.20, No.4, 287-299, 2001.

[10] M. M. Polycarpou. Fault accommodation of a class of multivariable nonlinear dy namical sy stems using a learning approach, IEEE Transactions on Automatic Control, Vol.46, No.5, 736-742, 2001.

[11] L. Xing, C. Wen, Z. Liu, H. Su, and J. Cai. Adaptive compensation for actuator failures with event-triggered input, Automatica, Vol.85, 129-136, 2017.

[12] W. Wang and C. Wen. Adaptive compensation for infinite number of actuator failures or faults, Automatica, Vol.47, 2197-2210, 2011.

[13] Q. Yang, S. S. Ge, and Y. Sun. Adaptive actuator fault tolerant control for uncertain nonlinear systems with multiple actuators, Automatica, Vol.60, 92-99, 2015.

[14] W. Wang and C. Wen. An open issue in direct adaptive solutions to actuator failure compensation problems, 5th IEEE Conference on Industrial Electronics and Applications, 127-130, 2010.

[15] A. Freddi, S. Longhi, A. Monteriù, D. Ortenzi, and D. Proietti Pagnotta. Fault Tolerant Control Scheme for Robotic Manipulators Affected by Torque Faults, IFAC-Papers OnLine, Vol.51, No.24, 886-893, 2018.

[16] H.-J. Ma and G.-H. Yang. Simultaneous fault diagnosis for robot manipulators with actuator and sensor faults, Information Sciences, Vol.366, 12-30, 2016.

[17] S. J. Yoo. Actuator fault detection and adaptive accommodation control of flexible-joint robots, IET Control Theory \& Applications, Vol.6, No.10, 1497-1507, 2012.

[18] Y. Zeng, Y.-R. Xing, H.-J. Ma, and G.-H. Yang. Adaptive fault diagnosis for robot manipulators with multiple actuator and sensor faults, The 27th Chinese Control and Decision Conference, Vol.366, 6569-6574, 2015.

[19] W. Gao and R. R. Selmic. Neural Network Control of a Class of Nonlinear Systems With Actuator Saturation, IEEE Transactions on Neural Networks, Vol.17, No.1, 147-156, 2006.

[20] J. M. Gomes da Silva, S. Tarbouriech, and G. Garcia. Local stabilization of linear systems under amplitude and rate saturating actuators, IEEE Transactions on Automatic Control, Vol.48, No.5, 842-847, 2003.

[21] X. Meng and L. Zhang. Analysis of a class of nonlinear systems in the presence of actuator saturation, 2009 Chinese Control and Decision Conference, 438-440, 2009.

[22] Z. Liu, J. Liu, and W. He. Partial differential equation boundary control of a flexible manipulator with input saturation, International Journal of Systems Science, Vol.48, No.1, 53-62, 2017. 